\section{Paying for high-energy physics}

SIR - Your article "Paying for highenergy physics" (Nature 1 December 1983, p.421) gives numerous false impressions.

The argument that it was not really necessary to observe the $\mathrm{W}^{ \pm}$and $\mathrm{Z}^{\circ}$ bosons, because "the [Salam-Weinberg] theory [of weak interactions] . . may . . . have been held to have been confirmed by what was learned (at CERN) in the 1970s about neutral currents" is wrong. The neutral current data, in common with all earlier information about weak interactions, are consistent with a force of zero range. It is true that those data are also consistent with the Glashow-Weinberg-Salam (GWS) theory, in which the weak interaction is due to the exchange of the $\mathrm{W}$ and $\mathrm{Z}$ bosons, thereby having finite range $\hbar / m_{\mathrm{Z}} c \sim 2 \times$ $10^{-16} \mathrm{~cm}$. However, the existence of such bosons was only a speculation until they were discovered at CERN earlier this year. The theoretical arguments for the existence of some structure in weak interactions at short distances were rather compelling but the GWS theory was not unique in fitting the earlier data. There were rival theories of the same type with additional bosons, and also very different theories which predicted $\mathrm{W}$ and $\mathrm{Z}$ bosons but as composite rather than fundamental objects.

The fact that the masses of the $\mathrm{W}$ and $\mathrm{Z}$ agree with the GWS predictions, within the errors, is certainly not sufficient to establish their true nature. Indeed, the experiments have reported some tentative evidence for the decay $\mathrm{Z}^{0} \rightarrow \mathrm{e}^{+} \mathrm{e}^{-} \gamma$, which cannot be explained by the GWS model. If confirmed, this radiative $Z^{0}$ decay might perhaps be accommodated by models in which the $\mathrm{W}$ and $\mathrm{Z}$ are composite. In any case, these anomalous events may indicate quite new physics to come. The fact that the GWS model is still potentially so vulnerable shows that we are still at the beginning of this chapter of the story.

Next, the 2,500 university physicists who use CERN facilities each year go there because it is their deep wish to be involved. There would be many more if there were funds to support them. It must also be remembered that this subject holds a very deep fascination both for the general public and especially for the young scientist still at school, as becomes very clear from interviewing for university entrance. What is going on at CERN is one of the most exhilarating enterprises mankind has known, a great flight of the creative human spirit to determine in full detail what a strange world we live in, and it is not surprising that so many physicists wish so profoundly to take part in this demanding work. You ask what they would be doing if CERN were not there: the answer is simply - "something less significant and much less interesting".

The insinuation that the advanced techniques used at CERN are just borrowed from American laboratories is simply false.
The proposal to transform the existing CERN Super Proton Synchrotron into the Proton-Antiproton Collider, with which the $\mathrm{W}$ and $\mathrm{Z}$ were produced, provides the most obvious counter-example. The crucial technique of stochastic cooling of the antiproton bunches - the breakthrough which made this step to much higher energies possible - was proposed and developed at CERN: it has now been taken up by the Americans for their Tevatron collider under construction at Fermilab, near Chicago. CERN also proposed and built the first proton-proton collider (ISR). Furthermore, CERN is notable for its development of new experimental methods; the neutrino horn, multiwire proportional drift chambers, the development and use of large liquid argon and/or uranium calorimeters, the ringimaging Cerenkov detector and the ISIS detector (a trend-setter in the problem field of particle identification at high energies) were all pioneered at CERN, to mention just a few outstanding items. Under the pressure of experimental needs, substantial technical developments have been made at CERN which have proved beneficial outside in fields as diverse as electronics and shipbuilding. It would be healthy for this country if more UK engineers were to gain practical experience by taking part in the advanced engineering work going on at CERN all the time.

We agree that the time may be ripe for intercontinental collaboration in the 1990 s. For the present, the Americans feel they may be able to build a superconducting collider on their own. However, we cannot anticipate any time when experiments will be unnecessary; "cheap theory" alone will not be able to account with certainty for the microscopic structure of the world in which we live, without the aid of experiment.

\section{R. H. DALITZ}

C. H. LLEWELLYN SMITH

Department of Theoretical Physics,

\section{University of Oxford,} Oxford OXI 3NP, UK

SIR - The "News and Views" item "Paying for high-energy physics"' (Nature 1 December 1983, p.421) contains so many inaccuracies that it is difficult to know how to reply adequately at reasonable length, but perhaps I may attempt to correct some of its more misleading assertions.

Comparing the SF 680 million CERN annual budget with the materials cost of the new electron-positron collider, LEP, is not comparing like with like. The annual budget is divided almost equally between staff costs - and many of the staff are engaged in building LEP at present - and materials costs. The materials budget this year is being spent on running five accelerators (one of which operates in two very different modes), and in building LEP. Electricity consumption is appreciable, but not outrageous for a large scientific laboratory whose facilities operate round the clock, seven days per week for much of the year. Indeed, it has been estimated recently that stopping all of CERN's accelerators for a complete year would save a total of just SF 25 million in electricity charges 3.7 per cent of the annual budget.

The super proton synchrotron will not be closed when LEP is completed. It is required as an injector for LEP and, more importantly, is expected to be used in the LEP era by up to half of the European high energy physics community for both fixed target and colliding beam experiments.

It is both scientifically unsound and unnecessarily churlish to attempt to run down CERN's recent triumphs in detecting the $\mathrm{W}$ and $\mathrm{Z}$ bosons. Maintaining that the observation of neutral currents by CERN a decade ago was a sufficient test of weak interaction theory is as sensible as claiming that the Venus de Milo may be adequately appreciated by studying the shadows cast on the floor by the spotlights that illuminate it. Continuing the analogy, the recent experiments have provided us with a clear silhouette, in remarkable agreement with the descriptions furnished by those distinguished intellectual tourists, Glashow, Salam and Weinberg. We are almost persuaded that they have been telling us the whole truth, but LEP should provide the detailed colour photographs that will settle the issue beyond doubt.

The article poses the question of what CERN's 2,500 visiting scientists would be doing if CERN did not exist. The most plausible answer is: "Working around the world in groups too small to be effective, lacking contact with their peers and the stimulation of direct competition, performing second-rate research with inadequate facilities". $\quad$ D. C. IMRIE Chairman,

SERC Sub-Committee on CERN, Department of Physics and Astronomy, University College London, London WCIE 6BT, UK

\section{No thanks - Anon}

SIR - Here is a formal "You're welcome" to all those authors who have recently included in the Acknowledgements section of their published papers a line thanking me, an Anonymous Reviewer, for critical comments on their manuscript. I suggest, however, a moratorium on this practice, since thanking me in print is a not particularly informative use of expensive journal space. If authors really feel that they must show their appreciation for an exceptionally helpful anonymous review, they can send me a personal note of thanks via journal editors. Then at least I'll know that it was my comments, and not those of some other Anonymous Reviewer, that were so appreciated. Name withheld by request - Nature's policy is not to thank Anonymous Reviewers - when the misdeed is spotted. 